\section{Zweitlinientherapie bei MDS}

Wirksame Zweitlinientherapien fehlen derzeit bei myelodysplastischen Syndromen (MDS) mit hohem Risiko. In einer kontrollierten Studie wurde die Effizienz des Multikinasehemmers Rigosertib getestet.

A ls hohes Risiko bei MDS gelten ein Anteil von 5-30\% Blasten im Knochenmark ohne effektive Behandlung und eine Lebenserwartung von unter 6 Monaten. Derzeitiger Standard sind hier hypomethylierende Agenzien (HMA) wie Azacitidin, die das Überleben verlängern. Versagen sie, sind die Aussichten schlecht, weil wirksame Zweitlinientherapien fehlen. Das mediane Überleben beträgt dann 4-6 Monate.

In die randomisierte Studie wurden 299 Patienten mit refraktärer Anämie mit Blastenexzess (RAEB-1 oder RAEB-2), RAEB in Transformation (RAEB- $t$ ) oder chronischer myelomonozytärer Leukä- mie eingeschlossen, bei denen in den beiden Jahren vor Studieneintritt eine HMATherapie versagt hatte. Die Patienten erhielten im Verhältnis 2:1 entweder Rigosertib $(1.800 \mathrm{mg} / \mathrm{Tag}$ kontinuierlich an den Tagen 1, 2 und 3, q2w) oder Best Supportive Care (BSC) mit oder ohne niedrigdosiertes Cytarabin. Nach 8 Zyklen wurde die Therapie mit Rigosertib auf 4-Wochen-Zyklen ( $\mathrm{q} 4 \mathrm{w}$ ) gestreckt. Das mediane Follow-up betrug 19,5 Monate.

Das mediane Gesamtüberleben war unter Rigosertib mit 8,2 Monaten nicht signifikant besser als unter BSC mit 5,9 Monaten (Hazard Ratio 0,87; p = 0,33). Post-hoc-Analysen legen allerdings nahe, dass bestimmte Subgruppen dennoch von der Therapie profitieren. Die häufigsten Nebenwirkungen vom Grad $\geq 3$ waren Anämie (18\% unter Rigosertib vs. $8 \%$ unter BSC), Thrombozytopenie (19 vs. $7 \%$ ), Neutropenie (17 vs. $8 \%$ ), febrile Neutropenie (12 vs. $11 \%$ ) und Pneumonie (12 vs. $11 \%$ ).

Fazit: Rigosertib verbesserte bei MDSPatienten nach Versagen einer HMATherapie das Überleben gegenüber BSC nicht signifikant. Derzeit wird in einer Phase-III-Studie geprüft, ob Subgruppen mit hohem Risiko einen Nutzen durch die Rigosertib-Therapie haben.

Kathrin von Kieseritzky

Garcia-Manero $\mathrm{G}$ et al. Rigosertib versus best supportive care for patients with high-risk myelodysplastic syndromes after failure of hypomethylating drugs (ONTIME): a randomised, controlled, phase 3 trial. Lancet Oncol. 2016;17(4):496-508.

\section{MDS: Risikoscore für Stammzelltransplantation}

Für Patienten mit myelodysplastischem Syndrom (MDS) ist eine allogene Stammzelltransplantation (alloSCT) die einzige kurative Therapieoption. Mit einem neuen Risikoscore hofft man, die Prognose nach alloSCT besser abschätzen zu können.

ie alloSCT birgt immer noch erhebliche Risiken und ihre Erfolgsaussichten sind von krankheits- und pati- entenbedingten Faktoren abhängig. Für den neuen Score wurden Daten von 2.133 MDS-Patienten analysiert, bei denen zwischen 2000 und 2012 eine alloSCT von HLA-gematchten $(\mathrm{n}=1.728)$ oder nicht gematchten Spendern $(\mathrm{n}=405)$ durchgeführt wurde.

In einer Trainingskohorte (1.151 Personen der HLA-gematchten Kohorte) wurde ein gewichteter Score erstellt, der bei den übrigen Patienten getestet wurde. Folgende Faktoren wurden berücksichtigt: Blasten $>3 \%$, Thrombozyten
Tab. 1: Überleben je nach Prognosescore bei HLA-gematchten Patienten

\begin{tabular}{|c|c|c|c|c|c|c|}
\hline \multirow[b]{2}{*}{ Risikogruppe } & \multicolumn{3}{|c|}{ Trainingskohorte } & \multicolumn{3}{|c|}{ Validierungskohorte } \\
\hline & n & HR (95\%-KI) & p-Wert & n & HR (95\%-KI) & p-Wert \\
\hline Niedrig (0-1) & 98 & 1,00 & & 47 & 1,00 & \\
\hline Intermediär (2-3) & 459 & $1,76(1,24-2,49)$ & 0,0017 & 258 & $1,66(1,01-2,71)$ & 0,045 \\
\hline Hoch (4-5) & 237 & $2,87(1,99-4,14)$ & $<0,001$ & 104 & $2,29(1,35-3,87)$ & 0,002 \\
\hline Sehr hoch $(\geq 6)$ & 45 & $6,75(4,28-10,67)$ & $<0,001$ & 18 & $5,02(2,48-10,15)$ & $<0,001$ \\
\hline Gesamt p-Wert & & & & & & $<0,001$ \\
\hline
\end{tabular}

$\leq 0 \times 10^{9} / 1$ bei Transplantation, Karnofsky-Performancestatus $<90 \%$, schlechtes oder sehr schlechtes zytogenetisches Risiko und Lebensalter 30-49 Jahre mit je 1 Punkt, monosomaler Karyotyp und Lebensalter $\geq 50$ Jahre mit je 2 Punkten.

In der HLA-gematchten Kohorte war das Scoringsystem mit dem Gesamtüberleben (OS) assoziiert (Tab. 1). Die Rate für das 3-Jahres-OS nach Transplantation betrug in der HLA-gematchten Validierungskohorte bei niedrigem Score (0-1 Punkt) $71 \%$, intermediärem (2-3 Punkte) $49 \%$, hohem (4-5 Punkte) $41 \%$ und sehr hohem Score ( $\geq 6$ Punkte) $25 \%(p<0,001)$. Mit dem Score stiegen in der HLA-gematchten Kohorte Rezidiv- $(\mathrm{p}<0,001)$ und therapiebedingtes Mortalitätsrisiko $(\mathrm{p}<0,001)$, in der nicht HLA-gematchten das Rezidivrisiko $(\mathrm{p}<0,001)$.

Fazit: Bei HLA-gematchter und bei nicht gematchter alloSCT erwies sich der Score als geeignetes prognostisches Hilfsmittel. Kathrin von Kieseritzky

Shaffer BC et al. Scoring System Prognostic of Outcome in Patients Undergoing Allogeneic Hematopoietic Cell Transplantation for Myelodysplastic Syndrome. J Clin Oncol. 2016; 34(16):1864-71. 